\title{
Electronic cigarette use and harm reversal: emerging evidence in the lung
}

Riccardo Polosa $a^{1,2,3}$

\begin{abstract}
Electronic cigarettes (ECS) have been rapidly gaining ground on conventional cigarettes due to their efficiency in ceasing or reducing tobacco consumption, competitive prices, and the perception of them being a much less harmful smoking alternative. Direct confirmation that long-term EC use leads to reductions in smoking-related diseases is not available and it will take a few decades before the tobacco harm reduction potential of this products is firmly established. Nonetheless, it is feasible to detect early changes in airway function and respiratory symptoms in smokers switching to e-vapor. Acute investigations do not appear to support negative respiratory health outcomes in EC users and initial findings from long-term studies are supportive of a beneficial effect of EC use in relation to respiratory outcomes. The emerging evidence that EC use can reverse harm from tobacco smoking should be taken into consideration by regulatory authorities seeking to adopt proportional measures for the e-vapor category.
\end{abstract}

Keywords: E-cigarette, E-vapor products, Harm reversal, Lung function, Respiratory system, Smoking cessation, Tobacco harm reduction

\section{Background}

The electronic cigarette $(E C)$ has been rapidly gaining ground on conventional cigarettes and could surpass consumption of conventional cigarettes within the next decade, according to some prediction analyses [1]. The growing popularity of ECs proves that many adult smokers are keen on using an alternative technologic form of smoking to reduce cigarette consumption or quit smoking and to relieve tobacco withdrawal symptoms [2]. Data from internet surveys [2,3] and clinical trials $[4,5]$ have shown that ECs may help smokers quit or reduce their tobacco consumption. Moreover, the popularity of ECs appears to be associated with the fact that they can be used in many smoke-free areas, their prices are competitive, and they are perceived as a much less harmful smoking alternative $[3,6]$.

Vapor toxicology under normal conditions of use is by far less problematic than that of conventional cigarettes

\footnotetext{
Correspondence: polosa@unict.it

${ }^{1}$ Centro per la Prevenzione e Cura del Tabagismo (CPCT), Azienda Ospedaliero-Universitaria "Policlinico-Vittorio Emanuele", Università di Catania, Catania, Italy

${ }^{2}$ Dipartimento di Medicina Clinica e Sperimentale - Università di Catania, Azienda Ospedaliero-Universitaria "Policlinico-Vittorio Emanuele", Università di Catania, Catania, Italy

Full list of author information is available at the end of the article
}

[7], and exclusive EC users have significantly lower urine levels of tobacco smoke toxicants and carcinogens compared to cigarette smokers [8]. Thus, smokers completely switching to regular EC use are likely to gain significant health benefits.

Although a reduction in smoking-related diseases from long-term EC use can be inferred by the positive findings on Swedish snus (a tobacco harm reduction product consisting of refined oral tobacco which is low in nitrosamines) [9], direct confirmation is not available and it will take a few decades before a reduction in individual and population health outcomes due to the regular use of e-vapor products can be firmly established. Nonetheless, it is feasible to detect early changes in airway function and respiratory symptoms in smokers switching to e-vapor.

In this commentary, I discuss the emerging potential of ECs for harm reversal with a specific focus on the respiratory system.

\section{Health outcomes and the respiratory system}

The lung is the primary target of the harmful effects of several airborne pollutants and cigarette smoke. Likewise, considering that inhalation is the exposure mechanism for EC use, the respiratory system is also the

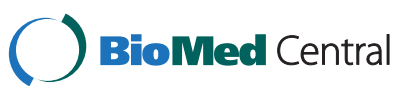


logical target for investigating any potential harmful effects of chemicals in the e-vapor.

Prospective clinical studies of well-characterized EC users would be the most informative to investigate respiratory health outcomes; however, such studies are quite demanding due to several methodological, logistical, ethical, and financial challenges. In particular, to address the potential of future disease development, hundreds of users would need to be followed for a substantial number of years before any conclusions could be made. A much less challenging approach is to explore cytotoxicity levels, mutagenicity, genotoxicity, oxidative stress, and inflammatory responses in human lung epithelial cell lines. However, these in vitro approaches also have inherent flaws; findings cannot be directly applied in human in vivo studies due to the inability to test the normal consumption exposure conditions of e-vapor products, the fact that standards for vapor production and exposure protocols have not been clearly defined, and the risk of over- or underestimating the interpretation of the toxic effects in these investigational models. Consequently, it is not surprising to find a divergence in the literature, with some authors reporting little or no injury [10], whereas others describing much higher level of toxicity and inflammatory responses despite using same cell lines [11]. Overall, despite the inconsistent and contradictory results, most acute in vitro studies are simply suggestive of non-specific irritant effects from evapor exposure. This is consistent with findings from internet surveys and clinical trials reporting transient throat irritation, dry cough, and other symptoms of respiratory irritation in some smokers when switching to ECs (reviewed in [7]).

Symptoms of irritation may occur in EC users hypersensitive to propylene glycol present in the e-vapor, but the possibility of unknown contaminants or byproducts contained in the e-vapor causing similar irritant effects cannot be discounted [12]. Likewise, a prompt defensive response against irritants from e-vapor inhalation is the most likely cause for the immediate physiologic changes detected with highly sensitive respiratory functional tests as reported by Vardavas et al. [13]. The question of whether such an irritation could translate into clinically meaningful lung disease remains unanswered, and there certainly is no evidence to date to suggest that there are any clinically significant adverse lung effects, at least acutely.

Moreover, it must be noted that the reported 16\% decrease in exhaled nitric oxide levels (i.e., $2.1 \mathrm{ppb}$ in absolute terms) and $11 \%$ increase in peripheral flow resistance by impulse oscillometry (i.e., $0.025 \mathrm{kPa} / \mathrm{L} / \mathrm{s}$ in absolute terms) from baseline after using an EC for 5 minutes were so small and well within test variability, that it is unlikely to have any clinical relevance
[14,15]. Moreover, no significant changes were detected by less sensitive respiratory function parameters (including forced expiratory volume in the first second (FEV1), forced vital capacity (FVC), FEV\% (FEV1/FVC index), and peak expiratory flow (PEF)) after EC use. Lack of a significant effect on exhaled nitric oxide and airflow obstruction as measured by FEV1, FVC, FEV\%, and PEF after short-term EC use has also been confirmed in a more recent study [16]. Finally, switching to EC use universally leads to a near-normalization in toxic levels of exhaled carbon monoxide (reviewed in [7]).

The very few studies mentioned above, which have focused on the acute effect of ECs on lung function, do not appear to support negative respiratory health outcomes in EC users. Nonetheless, only large and carefully conducted studies evaluating the long-term effects of these products will provide a definite answer regarding their impact on lung health.

As mentioned earlier, it would take hundreds of wellcharacterized EC users to be followed prospectively for a substantial number of years and very large funding to properly address the harm potential of ECs. At the University of Catania, we have structured an integrated clinical research program characterized by a minimalist approach entailing either highly sensitive respiratory functional tests to detect early changes of subclinical injury in 'healthy' smokers switching to EC or less sensitive but more robust respiratory function investigations to explore changes in EC users with preexisting lung disease. The initial findings are promising and generally supportive of a beneficial effect of EC use in relation to respiratory outcomes, both in health and disease.

Long-term changes in lung function have been monitored for up to 1 year in a large group of 'healthy' smokers who were invited to quit or reduce their tobacco consumption by switching to a first generation EC. Significant early positive changes from baseline of a sensitive measure of obstruction in the more peripheral airways (i.e., forced expiratory flow measured between $25 \%$ and $75 \%$ of FVC) were already detected at 3 months after switching in those who completely gave up tobacco smoking, with steady progressive improvements being observed also at 6 and 12 months (Polosa R, unpublished observation).

Asthma and chronic obstructive pulmonary disease (COPD) are progressive diseases characterized by persistent inflammatory and remodeling responses of the airways causing respiratory symptoms and progressive decline in lung function $[17,18]$. Although it is wellestablished that the inflammatory response to cigarette smoke plays a key role in COPD pathogenesis, increased morbidity and mortality have been reported in asthmatic individuals who smoke and quitting can significantly 
improve asthma symptoms and lung function [19]. Consequently, smokers with preexisting asthma and COPD may benefit from regular EC use. In the only clinical study conducted to ascertain efficacy and safety of EC use in asthma, substantial improvements in respiratory physiology and subjective asthma outcomes have been reported [20]. Exposure to e-vapor in this vulnerable population did not trigger any asthma attacks.

To date, no formal efficacy and safety assessment of EC use in COPD patients has been conducted. There is only evidence from a case series of three inveterate smokers with COPD, who eventually quit tobacco smoking on their own by switching to an EC [21]. Significant improvement in quality of life and reduction in the number of disease exacerbations were noted. EC use was well tolerated with no reported adverse events.

The reported improvements of respiratory patients who have become regular ECs users are consistent with findings from a large internet survey of regular EC users diagnosed with asthma and COPD [2]. An improvement in symptoms of asthma and COPD after switching was reported in $65.4 \%$ and $75.7 \%$ of the respondents, respectively. Compared to dual users, improvement in symptoms of asthma and COPD were more often reported by exclusive EC users. After switching, medications were stopped in 460/2,498 (18.4\%) respondents with asthma and COPD. Worsening after switching was only reported in $1.1 \%$ of the asthmatics and in $0.8 \%$ of the COPD respondents. Taken together, these findings provide emerging evidence that EC use can reverse harm from tobacco smoking.

\section{Conclusions and implications for policymaking}

Compared to combustible cigarettes, e-vapor products are at least $96 \%$ less harmful and may substantially reduce individual risk and population harm [22]. Future research will better define and further reduce residual risks from EC use to as low as possible by establishing appropriate quality control and standards. Although large longitudinal studies are warranted to elucidate whether ECs are a less harmful alternative to tobacco cigarettes and whether significant health benefits can be expected in smokers who switch from tobacco to ECs, the emerging evidence that EC use can reverse harm from tobacco smoking should be taken into consideration by regulatory authorities seeking to adopt proportional measures for the e-vapor category [23].

\footnotetext{
Abbreviations

COPD: Chronic obstructive pulmonary disease; ECs: Electronic cigarettes; FEV\%: FEV1/FVC index; FEV1: Forced expiratory volume in the first second; FVC: Forced Vital Capacity; PEF: Peak Expiratory Flow.
}

\section{Competing interests}

$\mathrm{RP}$ has received grant support from respiratory drug manufacturers including CV Therapeutics, NeuroSearch A/S, Sandoz, Merck Sharp \& Dohme, and
Boehringer-Ingelheim; he has served as a speaker for CV Therapeutics, Novartis, Merck Sharp \& Dohme, and Roche and as a consultant for CV Therapeutics, Duska Therapeutics, Neuro-Search A/S, Boehringer-Ingelheim, and Forest Laboratories. He has received payment for developing educational presentations from Merck Sharp \& Dohme, Novartis, and Almirall. $\mathrm{RP}$ has also received lecture fees and research funding from manufacturers of stop smoking medications including GlaxoSmithKline and Pfizer and served as a consultant for Pfizer, Global Health Alliance for treatment of tobacco dependence, Arbi Group Srl (an Italian e-cigarette distributor) and ECITA (Electronic Cigarette Industry Trade Association, in the UK).

\section{Author information}

$\mathrm{RP}$ is Full Professor of Internal Medicine with tenure supported by the University of Catania, Italy. RP serves as scientific advisor for LIAF (acronym for Italian Anti-Smoking League).

\section{Acknowledgments}

RP's research on smoking, electronic cigarettes, and asthma is currently supported by the University of Catania and LIAF (acronym for Italian Anti-Smoking League).

\section{Author details}

${ }^{1}$ Centro per la Prevenzione e Cura del Tabagismo (CPCT), Azienda Ospedaliero-Universitaria "Policlinico-Vittorio Emanuele", Università di Catania, Catania, Italy. ${ }^{2}$ Dipartimento di Medicina Clinica e Sperimentale - Università di Catania, Azienda Ospedaliero-Universitaria "Policlinico-Vittorio Emanuele", Università di Catania, Catania, Italy. ${ }^{3}$ UOC di Medicina Interna e d'Urgenza, Edificio 4, Piano 3, AOU "Policlinico-V. Emanuele", Via S. Sofia 78, 95123 Catania, Italy.

Received: 16 February 2015 Accepted: 17 February 2015

Published online: 18 March 2015

\section{References}

1. Mangan D. E-cigarette sales are smoking hot, set to hit $\$ 1.7$ billion. CNBC. 2013. Retrieved 28th August 2014 from http://www.cnbc.com/id/100991511.

2. Farsalinos KE, Romagna G, Tsiapras D, Kyrzopoulos S, Voudris V. Characteristics, perceived side effects and benefits of electronic cigarette use: a worldwide survey of more than 19,000 consumers. Int J Environ Res Public Health. 2014;11:4356-73.

3. Siegel MB, Tanwar KL, Wood KS. Electronic cigarettes as a smoking cessation tool: Results from an online survey. Am J Prev Med. 2011:40:472-5.

4. Caponnetto P, Campagna D, Cibella F, Morjaria JB, Caruso M, Russo C, et al EffiCiency and Safety of an eLectronic cigAreTte (ECLAT) as tobacco cigarettes substitute: a prospective 12-month randomized control design study. PLoS One. 2013;8:e66317.

5. Polosa R, Caponnetto P, Maglia M, Morjaria JB, Russo C. Success rates with nicotine personal vaporizers: a prospective 6-month pilot study of smokers not intending to quit. BMC Public Health. 2014;14:1159.

6. Biener L, Hargraves JL. A longitudinal study of electronic cigarette use among a population-based sample of adult smokers: association with smoking cessation and motivation to quit. Nicotine Tob Res. 2015;17:127-33.

7. Farsalinos KE, Polosa R. Safety evaluation and risk assessment of electronic cigarettes as tobacco cigarettes substitutes: a systematic review. Ther Adv Drug Safety. 2014;5:67-86.

8. Hecht SS, Carmella SG, Kotandeniya D, Pillsbury ME, Chen M, Ransom BW, et al. Evaluation of toxicant and carcinogen metabolites in the urine of e-Cigarette users versus cigarette smokers. Nicotine Tob Res. 2014; [Ahead of print].

9. Lee PN. Summary of the epidemiological evidence relating snus to health. Regul Toxicol Pharmacol. 2011;59:197-214

10. Misra M, Leverette RD, Cooper BT, Bennett MB, Brown SE. Comparative in vitro toxicity profile of electronic and tobacco cigarettes, smokeless tobacco and nicotine replacement therapy products: e-liquids, extracts and collected aerosols. Int J Environ Res Public Health. 2014;11:11325-47.

11. Cervellati F, Muresan XM, Sticozzi C, Gambari R, Montagner G, Forman HJ, et al. Comparative effects between electronic and cigarette smoke in human keratinocytes and epithelial lung cells. Toxicol In Vitro. 2014;28:999-1005.

12. Polosa R, Campagna D, Tashkin D. Subacute bronchial toxicity induced by an electronic cigarette: take home message. Thorax. 2014;69:588. 
13. Vardavas C, Anagnostopoulos N, Kougias M, Evangelopoulou V, Connolly G, Behrakis P. Short-term pulmonary effects of using an electronic cigarette: impact on respiratory flow resistance, impedance, and exhaled nitric oxide. Chest. 2012;141:1400-6.

14. Oostveen E, MacLeod D, Lorino H, Farré R, Hantos Z, Desager $K$, et al. The forced oscillation technique in clinical practice: methodology, recommendations and future developments. Eur Respir J. 2003;22:1026-41.

15. Dweik RA, Boggs PB, Erzurum SC, Irvin CG, Leigh MW, Lundberg JO, et al. An official ATS clinical practice guideline: interpretation of exhaled nitric oxide levels (FENO) for clinical applications. Am J Respir Crit Care Med. 2011;184:602-15

16. Flouris A, Chorti M, Poulianiti K, Jamurtas A, Kostikas K, Tzatzarakis M, et al. Acute impact of active and passive electronic cigarette smoking on serum cotinine and lung function. Inhal Toxicol. 2013;25:91-101.

17. Morjaria JB, Malerba M, Polosa R. Biologic and pharmacologic therapies in clinical development for the inflammatory response in COPD. Drug Discov Today. 2010;15:396-405.

18. Holgate ST, Polosa R. Treatment strategies for allergy and asthma. Nat Rev Immunol. 2008;8:218-30.

19. Polosa R, Thomson NC. Smoking and asthma: dangerous liaisons. Eur Respir J. 2013;41:716-26.

20. Polosa R, Morjaria JB, Caponnetto P, Caruso M, Strano S, Battaglia E, et al. Effect of smoking abstinence and reduction in asthmatic smokers switching to electronic cigarettes: evidence for harm reversal. Int J Environ Res Public Health. 2014;11:4965-77.

21. Caponnetto P, Polosa R, Russo C, Leotta C, Campagna D. Successful smoking cessation with electronic cigarettes in smokers with a documented history of recurring relapses: a case series. J Med Case Rep. 2011;5:585.

22. Nutt DJ, Phillips LD, Balfour D, Curran HV, Dockrell M, Foulds J, et al. Estimating the harms of nicotine-containing products using the MCDA approach. Eur Addict Res. 2014;20:218-25.

23. Saitta D, Ferro GA, Polosa R. Achieving appropriate regulations for electronic cigarettes. Ther Adv Chronic Dis. 2014;5:50-61.

\section{Submit your next manuscript to BioMed Central and take full advantage of:}

- Convenient online submission

- Thorough peer review

- No space constraints or color figure charges

- Immediate publication on acceptance

- Inclusion in PubMed, CAS, Scopus and Google Scholar

- Research which is freely available for redistribution 